\title{
Incidence of Graft Detachment Compared Between Two Techniques of Ultrathin Descemet's Stripping Automated Endothelial Keratoplasty: A Case Series.
}

Oren Mark Feuerman ( $\nabla$ oren.feuerman@gmail.com )

Rovigo Hospital: Ospedale Santa Maria della Misericordia https://orcid.org/0000-0001-6039-023X

Luigi Caretti

Rovigo Hospital: Ospedale Santa Maria della Misericordia

Antonio Agresta

Rovigo Hospital: Ospedale Santa Maria della Misericordia

Cristina Monterosso

Angel Hospital: Ospedale dell'Angelo-Mestre

Giacomo Verzola

Rovigo Hospital: Ospedale Santa Maria della Misericordia

\section{Research Article}

Keywords: dsaek, ut-dsaek, rebubbling, graft survival, graft dislocation

Posted Date: January 28th, 2022

DOI: https://doi.org/10.21203/rs.3.rs-1284448/v1

License: (c) (i) This work is licensed under a Creative Commons Attribution 4.0 International License.

Read Full License 


\section{Abstract \\ Purpose}

To evaluate the incidence of rebubbling for graft detachment in two different surgical techniques and postoperative management of Ultrathin Descemet's Stripping Automated Endothelial Keratoplasty (UTDSAEK).

\section{Methods}

A Longitudinal retrospective and prospective case series of 50 eyes operated between November $1 \mathrm{st}$, 2017 and November 1st, 2020. Graft detachment and consequent rebubbling incidence were registered and reviewed in two groups of consecutive patients with Fuchs endothelial dystrophy. The the two surgical techniques differed in diameter of Descemetorhexis and presence or absence of preoperative inferior Nd:YAG laser iridotomy. Main outcome measures were incidence of rebubbling in 30 days postoperative period and incidence of ocular hypertention.

\section{Results}

Incidence of graft detachment and consequent rebubbling procedure are lower among eyes who underwent iridotomy and had a larger diameter Descemetorhexis than graft diameter. Intraocular pressure resulted higher among patients who did not undergo preoperative inferior Nd:YAG laser iridotomy.

\section{Conclusions}

Preoperative lower iridotomy and less postoperative eye manipulation are associated with less graft dislocation following Ultrathin Descemet's Stripping Automated Endothelial Keratoplasty.

\section{Introduction:}

Descemet's Stripping Automated Endothelial Keratoplasty (DSAEK) is a relatively new surgical technique introduced just 15 years ago by Price and Price and M. Govory[1, 2]. Ever since its first introduction numerous clinical trials have demonstrated the clinical safety and efficacy in treating endothelial corneal disorders. A more recent evolution of DSAEK is the Ultrathin DSAEK, firstly described by M. Busin et al in 2012[3]. UT-DSAEK may be considered as a bridge technique between standard DSAEK and Descemet's Membrane Endothelial Keratoplasty (DMEK)[4]. The main difference between DSAEK and UT-DSAEK is the graft thickness (100 microns or thinner), obtained with the microkeratome double-pass technique. A very recent large retrospective case series of over 300 eyes showed excellent 5-year outcomes, in terms of BCVA, endothelial cell density and survival rates of the graft, comparable with those recorded postDMEK[5]. Furthermore, the most frequent early post-operative complication, which is graft dislocation or 
detachment, is not higher among patients who underwent UT-DSAEK[5-7]. When indicated, UT-DSAEK is undoubtedly a more suitable surgical technique respect to a full thickness penetrating keratoplasty, resulting in a better anatomical and functional outcome and having less intra and post-operative severe complications.

\section{Methods:}

Phakic or pseudophakic adult patients with endothelial dysfunction were enrolled in the case series. Exclusion criteria included presence of tube shunts in anterior chamber, anterior chamber intraocular lenses, diffuse anterior synechiae and uncontrolled glaucoma. Prior to surgery a thorough eye examination and medical and ophthalmological history registration were performed. An informed consent was obtained from all patients according to the Declaration of Helsinki seventh revision of 2013. Surgery was performed by a single experienced surgeon between November 1st, 2017 and November 1st, 2020 in the Ophthalmology Unit of the Rovigo Public Hospital in Italy. Following the procedure all patients were evaluated at 6 hours, 24 hours, 1 week and one month after surgery. Management of moderate ocular hypertension (defined as measured IOP $\geq 25 \mathrm{mmHg}$ with Goldmann Applanation tonometry), detected in the 6 hours post-surgery evaluation, differed between the two groups of patients, in group $A$, included 30 eyes without preoperative inferior YAG:Nd laser iridotomy and smaller diameter Descemetorhexis, manual evacuation of the air bubble was performed at the slit lamp, applying gentle pressure with the tip of a $30 \mathrm{G}$ needle on the distal part of the secondary incision, leaving at least $30 \%$ of the air bubble in the anterior chamber, whereas in the other group, B, included 20 eyes with preoperative lower Nd:YAG laser iridotomy performed 72 hours prior to surgery and diameter Descemetorhexis, moderate ocular hypertension was managed with systemic IOP lowering drugs, thus avoiding any manual manipulation of the operated eye. Graft dislocation defined as fluid interface of an otherwise well positioning graft, as well as complete dislocation into the anterior chamber[6], was detected by slit lamp biomicroscopy and further confirmed by AS OCT (CASIA 2, Tomey GMBH, Nürnberg, Germany). Any graft detachment detected at 24 hours or within 7 days post-surgery, was reattached with rebubbling under topical anesthesia using a $30 \mathrm{G}$ needle in the operation room. Regardless of graft status, topical antibiotic-corticosteroid eye drops four times a day for 30 days was prescribed after surgery and were tapered down accordingly.

Surgical Techniques:

All of the surgeries were performed at the 12-o'clock position, under local anesthesia, by a single experienced surgeon (L.C) with corneal grafts obtained from the Fondazione banca Degli Occhi del Veneto Onlus, Mestre, Italy (Veneto Eye Bank Foundation) using the microkeratome assisted double pass technique[8]. The precut donor graft was trephined to an $8.50 \mathrm{~mm}$ diameter using the Moria Guarded punch (Moria, Antony, France). Phakic patients underwent a standard microincisional phacoemulsification and a single piece IOL implant whereas pseudophakic patient's recipient eyes were prepared directly for the tissue graft. Paralimbal clear cornea incision were performed with a $15^{\circ}$ single use blade (Alcon Surgical, Fort Worth, TX, USA) for an anterior chamber maintainer, service incision and 
the $3.5 \mathrm{~mm}$ long main incision for the insertion of the tip of the Busin glide (Moria, Antony, France). In phakic eyes, under a cohesive OVD (Ophthalmic Viscosurgical Device) (Healon; Abbott Medical, Uppsala, Sweden), a Descemet membrane and endothelim removal was performed using a reversed PriceeSinskey hook (Moria, Antony, France) to a diameter of $8.50 \mathrm{~mm}$ in the group A patient's eye and to a slightly larger diameter of $8.75 \mathrm{~mm}$ in the group B patient's eye. When OVD was used, the surgeon reassured a complete removal of the latter from the anterior chamber prior to insertion of the donor graft, using the pull-through technique, followed by a filtered air tamponade to achieve a complete filling of the anterior chamber volume. In phakic eyes Descemetorhexis and graft insertion were performed exclusively under BSS (Balanced Salt Solution, Puri Clear, Zeiss Meditec AG, Germany). After surgery, regardless of the patient groups, all patients were sent to a 6 -hour period of observation, maintaining a face up position. When graft detachment was detected 24 hours or within 7 days post-surgically, a rebubbling procedure was performed under topical anesthesia using a $30 \mathrm{G}$ needle in the operation room.

\section{Statistical analysis:}

Demographics, surgery type and graft characteristics, parametric and non-parametric continuous variables were expressed by mean \pm standard deviation (SD) and median with interquartile range (IQR), respectively. In order to detect predictors of rebubbling we analyzed the following variables: Patient age (years), IOP values at 6 and 24 hours, 1 week and 1 month, postoperatively, patient sex, graft characteristics and the two surgical techniques. Comparison of continuous variables with a parametric and non-parametric distribution were performed using T-test and Wilcoxon's rank-sum test, respectively. Pearson's chi square (or Fisher's exact test when appropriate) was used to evaluate bivariate associations between categorical variables. Significance was set at $5 \%$. All analysis were assessed by using R studio v. 1.3.1073.

\section{Results:}

A total number of 50 patients underwent UT-DSAEK surgery between November 1st, 2017 and November 1 st, 2020 by a single expert corneal surgeon. 30 eyes underwent surgery using technique $A$, whereas 20 eyes underwent surgery using technique $B$ (see methods paragraph for further details). Overall, group A and group $B$ treated patients had a median age of $75.3 \pm 7.3$ years, $76.8 \pm 6.9$ years and $73.8 \pm 7.8$ years, respectively. Indications for surgery in the whole cohort, in group A and group B were endothelial dystrophy in $80 \%, 77 \%$ and $85 \%$, respectively, pseudophakic bullous keratopathy in $12 \%, 13 \%$ and $10 \%$, respectively and previous DSAEK surgery failure in $8 \%, 10 \%$ and $5 \%$, respectively. Preoperative eye status was phakic in $84 \%, 83 \%$ and $85 \%$, among all patients, group A and group B treated patients, respectively (see Table 1 for full demographic data) For all the patients, corneal grafts were obtained from donors aged $68 \pm 10$ years with an endothelial cell density of $2433 \pm 199$ cells $/ \mathrm{mm}^{2}$ and delivered by the Eye bank with an average thickness of $89 \pm 9$ microns (Table 2). Moderate ocular hypertension that required a manual air bubble evacuation from anterior chamber was verified in 7 out of 30 eyes among group $A$ patients (23.3\%) and in none of the group B eyes. 
Table 1

Demographics

\begin{tabular}{|llll|}
\hline & Overall & Tecniche A & Tecniche B \\
\hline Total number of Eyes/Patients & 50 & 30 & 20 \\
\hline Age (yrs), mean ( \pm SD) & $75.3 \pm(7.3)$ & $76.8 \pm(6.9)$ & $73.8 \pm(7.8)$ \\
\hline Sex (women/men) N & $31 / 19$ & $18 / 12$ & $13 / 7$ \\
\hline Indications for UT-DSAEK N (\%) & - & - & - \\
\hline Endothelial dystrophy & $40(80 \%)$ & $23(77 \%)$ & $17(85 \%)$ \\
\hline Pseudophakic bollous keratopathy & $6(12 \%)$ & $4(13 \%)$ & $2(10 \%)$ \\
\hline Previous DSAEK faliure & $4(8 \%)$ & $3(10 \%)$ & $1(5 \%)$ \\
\hline Preoperative status N (\%) & - & - & - \\
\hline Phakic & $42(84 \%)$ & $25(83 \%)$ & $17(85 \%)$ \\
\hline Pseudophakic & $8(16 \%)$ & $5(17 \%)$ & $3(15 \%)$ \\
\hline Legend to Table 1: SD: Standard Deviation. & & \\
\hline
\end{tabular}

Table 2

Graft characteristics

\begin{tabular}{|llll|}
\hline & Overall & Tecniche A & Tecniche B \\
\hline Total number of Eyes/Patients & 50 & 30 & 20 \\
\hline Donor age $(\mathrm{yrs})( \pm \mathrm{SD})$ & $68 \pm(10)$ & $69 \pm(9)$ & $66 \pm(12)$ \\
\hline ECD (cells/mm²) $( \pm \mathrm{SD})$ & $2433 \pm(199)$ & $2423 \pm(224)$ & $2448 \pm(159)$ \\
\hline Thickness (microns) $( \pm$ SD $)$ & $89 \pm(9)$ & $87 \pm(11)$ & $91 \pm(7)$ \\
\hline \multicolumn{2}{|l}{ Legend to Table 2: SD: Standard Deviation. } & & \\
\hline
\end{tabular}

Among all the median IOP values measured postoperatively, only the 6 hours median IOP was statistically higher among group A patients, $17.5 \mathrm{mmHg}(14-24)$ versus $15 \mathrm{mmHg}(13.75$ - 16.5) with $\mathrm{p}$ value of 0.04 . Time to rebubbling was not statistically different between group $A$ and group $B$ with median values of 2 days (2-3) and 2.7 days (2-3), p value 0.73 , respectively. Endothelial graft dislocation and consequent rebubbling was significantly higher among group A patients, respect to group $B$ patients, 17 out of $30(56.7 \%)$ versus 4 out of $20(20 \%)$, p value of 0.02 , respectively (Table 3 ). 
Table 3

Univariate Analysis for Rebubbling Predictors Assessment

\begin{tabular}{|c|c|c|c|}
\hline & Tecniche A & Tecniche B & $P$ value \\
\hline Eyes/Patients & 30 & 20 & - \\
\hline Sex (women/men) & $18 / 12$ & $13 / 7$ & 0.95 \\
\hline Age (yrs), mean ( $\pm S D)$ & $76.8 \pm(6.9)$ & $73.8 \pm(7.8)$ & 0.15 \\
\hline IOP (mmHg), median (IQR) & & & - \\
\hline 6 hours & $17.5(14-24)$ & $15(13.75-16.5)$ & 0.04 \\
\hline 24 hours & $15(13-20)$ & $13.5(12-16)$ & 0.22 \\
\hline 1 week & $14(13-16.75)$ & $14(12-16)$ & 0.53 \\
\hline 1 month & $14.5(12.25-17.5)$ & $15(12.75-17.25)$ & 0.70 \\
\hline Time to Rebubbling in days, median (IQR) & $2.0(2.0-3.0)$ & $2.5(2.0-3.0)$ & 0.73 \\
\hline Rebubbling, N (\%) & $17(56.7)$ & $4(20)$ & 0.02 \\
\hline
\end{tabular}

\section{Discussion:}

To date there is a lack of high-quality comparative studies regarding different surgical techniques and the associated complications and outcomes of endothelial corneal transplantation using ultrathin grafts. Endothelial transplantation using ultrathin grafts is the latest evolution of DSAEK and has been demonstrated excellent and effective as described by Busin M. et al[5], however different intra and perioperative factors and patient pre and postoperative preparation and management were not thoroughly evaluated by comparative studies. A recent high quality multicenter randomized clinical trial performed on 1330 eyes as a part of the Cornea Preservation Study Group (CPTS) in 2019, analyzed the association of donor, recipient, and operative factors on graft dislocation after DSAEK, as well as the effects of graft dislocation and elevated IOP on graft success 3 years postoperatively[9]. Among the factors associated with graft dislocation history of donor diabetes, increased pre-lamellar dissection central corneal thickness and operative complications were mostly associated with endothelial graft dislocation. Interestingly the CPTS described that acute elevation of IOP in the early postoperative period had a negative impact on graft success with more than 3-fold increased risk of failure probably due to endothelial cell density reduction induced by high IOP induced cell damage. Since donor tissue adherence is most likely dependent on a healthy endothelium that begins pumping fluid from the overlying cornea[10], endothelial disfunction clearly impact negatively on graft attachment. This study included a homogeneous population in terms of age, surgical indications, preoperative status 
(phakic/pseudophakic) and graft characteristics, making the comparative statistical analysis reliable. Based on the previous knowledge that less eye manipulation immediately after surgery is associated with less endothelial graft dislocation[1, 11], we decided to conduct a comparison between two surgical techniques, $A$ and $B$, in which postoperative ocular hypertension was managed with the least eye manipulation and air bubble evacuation from anterior chamber in the latter technique. We hypothesized that air bubble manual evacuation induces microturbulences in the aqueous humor and lowers the positive pressure that is exerted on the endothelial graft, increasing the risk of graft dislocation. Less eye manipulation was possible due to the presence of inferior iridotomy prior to surgery and the management of postoperative ocular hypertension with systemic IOP lowering drugs. Results from our study show a statistically significant lower IOP measured 6 hours postoperatively $(17.5 \mathrm{mmHg}$ vs. $15 \mathrm{mmHg}, \mathrm{p}=0.04)$ among group $B$ patients who underwent preoperative lower YAG laser iridotomy. Moreover, moderate postoperative ocular hypertension (IOP $\geq 25 \mathrm{mmHg}$ ) was present in 7 out of 30 eyes among patients who did non undergo inferior iridotomy, and in none of the patients who underwent inferior iridotomy. Different Descemet stripping diameter in the recipients also was included in our comparative study. An interesting paper by Romano et al. evaluated the influence of graft size on graft survival in DSAEK[12], finding that a larger graft diameter ( $9 \mathrm{~mm}$ and beyond) is associated with lower failure rate having approximately 10$20 \%$ more endothelial cells[13]. Maintaining an $8.50 \mathrm{~mm}$ diameter of the endothelial graft in all of our patients, we tried to determine whether a different Descemet stripping was associated with early graft dislocation. From the statistical analysis we could not find an association between different Descemet stripping diameter and graft dislocation; however, based on previous studies, it could be recommended to yield in the future for larger than $8.5 \mathrm{~mm}$ diameter endothelial graft in UT-DSAEK. In this study the incidence of endothelial graft dislocation and consequent rebubbling procedure was statistically lower among group $B$ patients $(p=0.02)$. Correlating the lower incidence of graft dislocation with the lower postoperative IOP, and thus less manual anterior chamber air evacuation, inferior preoperative YAG:Nd laser iridotomy is probably a procedure that improve the outcome of UT-DSAEK and lowers the postoperative IOP measured 6 hours after surgery. Among the other rebubbling predictors univariate assessment, there were no other statistically significant differences between the two techniques (Table 3). This study was conducted mainly to evaluate pre and intraoperative procedures associated with endothelial graft dislocation. For that reason, a relatively short follow up of 1 month was established. The relatively short follow up did not permit to evaluate other important outcomes such as the effect of rebubbling on visual acuity, endothelial cell density and graft transparency that is expected to be lower as previous studies showed that prolonged and repeated air exposure is toxic to the corneal endothelium[14]. In addition, a longer follow-up would have been useful to evaluate the long-term differences in IOP and also if the postoperative moderately high IOP rise seen in patients who did not undergo inferior YAG:Nd laser iridotomy, would have caused anatomical and functional damage to the optic nerve. Postoperative visual acuity was not evaluated in this comparative study as it is well known that a full recovery from UT-DSAEK surgery may take up to one year post surgically[5].

\section{Conclusions:}


Corneal endothelial transplant using ultrathin graft is an excellent bridging technique that features an optimal ratio between visual outcome and recovery time and technical complexity respect to DMEK. This case study demonstrates that early postoperative ocular hypertension is a probable negative predictive factor for graft dislocation and consequent rebubbling, thus preoperative inferior YAG:Nd laser iridotomy and less eye manipulation in the immediate post-operative period are strategies that lower the incidence of graft dislocation in ultrathin DSAEK. Conversely, different recipient Descemet stripping do not seem to influence the incident of early graft dislocation.

\section{Declarations}

Funding: The authors declare that no funds, grants, or other support were received during the preparation of this manuscript.

Competing Interests: The authors have no relevant financial or non-financial interests to disclose

Author Contributions: Dr. Luigi Caretti M.D, Dr. Oren Mark Feuerman M.D and Dr. Antonio Agresta M.D contributed to the study conception and design. Material preparation, data collection and analysis were performed by Dr. Antonio Agresta and Giacomo Verzola Orthoptist. The first draft of the manuscript was written by Dr. Oren Mark Feuerman. All authors read and approved the final manuscript.

Ethics approval: This study was performed in line with the principles of theDeclaration of Helsinki seventh revision of 2013.Approval was granted by the local Ethics Committee of the Public hospital of Rovigo, Italy (AziendaUlss 5 Polesana)

Consent to participate: Informed consent was obtained from all individual participants included in the study.

Consent to publish:The authors affirm that human research participants provided informed consent for publication of the images in Figure 1.

\section{References}

1. Gorovoy MS (2006) Descemet-stripping automated endothelial keratoplasty. Cornea. https://doi.org/10.1097/01.ico.0000214224.90743.01

2. Price FW, Price MO (2005) Descemet's stripping with endothelial keratoplasty in 50 eyes: A refractive neutral corneal transplant. J Refract Surg. https://doi.org/10.3928/1081-597x-20050701-07

3. Busin M, Patel AK, Scorcia V, Ponzin D (2012) Microkeratome-assisted preparation of ultrathin grafts for descemet stripping automated endothelial keratoplasty. Investig Ophthalmol Vis Sci. https://doi.org/10.1167/iovs.11-7753

4. Taravella MJ, Shah V, Davidson R (2013) Ultrathin DSAEK. Int Ophthalmol Clin. https://doi.org/10.1097/II0.0b013e31827823a8 
5. Madi S, Leon P, Nahum Y, et al (2019) Five-Year Outcomes of Ultrathin Descemet Stripping Automated Endothelial Keratoplasty. Cornea. https://doi.org/10.1097/IC0.0000000000001999

6. Lee WB, Jacobs DS, Musch DC, et al (2009) Descemet's Stripping Endothelial Keratoplasty: Safety and Outcomes. A Report by the American Academy of Ophthalmology. Ophthalmology. https://doi.org/10.1016/j.ophtha.2009.06.021

7. Price MO, Price FW (2010) Endothelial keratoplasty - a review. Clin. Exp. Ophthalmol.

8. Busin M, Madi S, Santorum P, et al (2013) Ultrathin descemet's stripping automated endothelial keratoplasty with the microkeratome double-pass technique: Two-year outcomes. Ophthalmology. https://doi.org/10.1016/j.ophtha.2012.11.030

9. Aldave AJ, Terry MA, Szczotka-Flynn LB, et al (2019) Effect of Graft Attachment Status and Intraocular Pressure on Descemet Stripping Automated Endothelial Keratoplasty Outcomes in the Cornea Preservation Time Study. Am J Ophthalmol. https://doi.org/10.1016/j.ajo.2019.02.029

10. Bhalerao S, Mohamed A, Vaddavalli P, et al (2020) Outcomes of rebubbling for graft detachment after Descemet's stripping endothelial keratoplasty or Descemet's stripping automated endothelial keratoplasty. Indian J Ophthalmol. https://doi.org/10.4103/ijo.IJO_1521_18

11. Price MO, Price FW (2006) Descemet's Stripping with Endothelial Keratoplasty. Comparative Outcomes with Microkeratome-Dissected and Manually Dissected Donor Tissue. Ophthalmology. https://doi.org/10.1016/j.ophtha.2006.05.034

12. Romano V, Tey A, Hill NME, et al (2015) Influence of graft size on graft survival following Descemet stripping automated endothelial keratoplasty. $\mathrm{Br} \mathrm{J}$ Ophthalmol. https://doi.org/10.1136/bjophthalmol-2014-305648

13. Amann J, Holley GP, Lee SB, Edelhauser HF (2003) Increased endothelial cell density in the paracentral and peripheral regions of the human cornea. Am J Ophthalmol. https://doi.org/10.1016/S0002-9394(02)02237-7

14. Kopsachilis N, Tsaousis KT, Tsinopoulos IT, Welge-Luessen U (2013) Air toxicity for primary humancultured corneal endothelial cells: An in vitro model. Cornea. https://doi.org/10.1097/ICO.0b013e31826895f8.

\section{Figures}




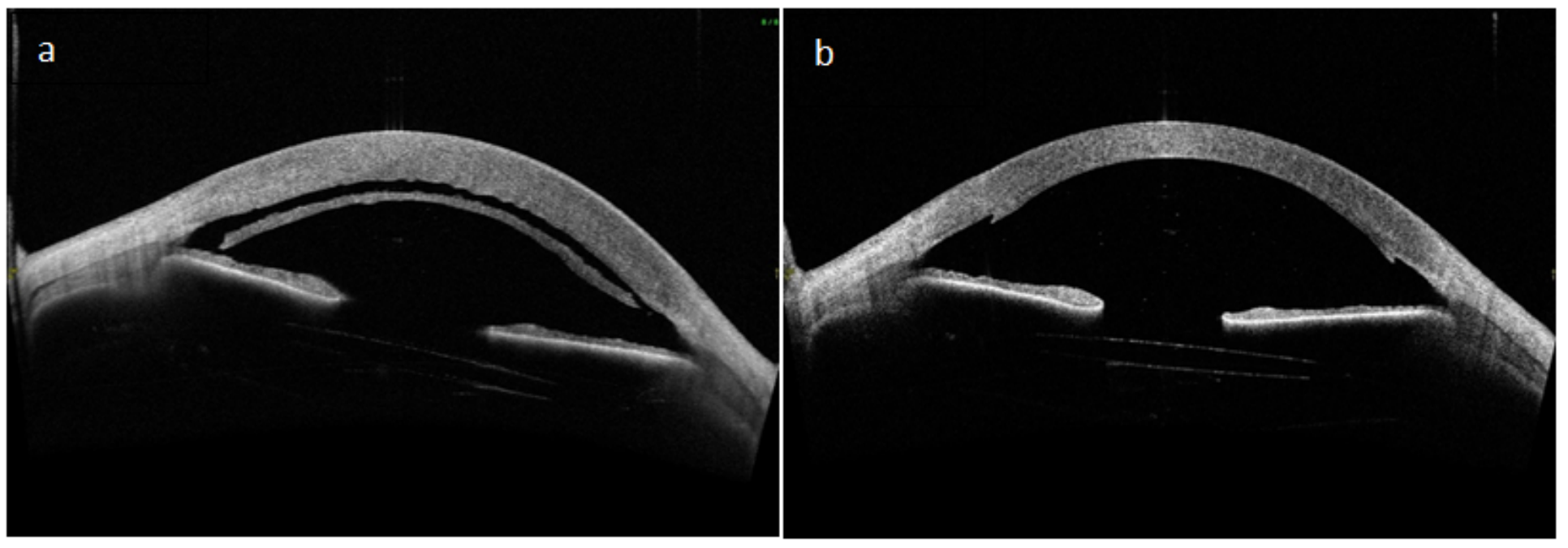

Figure 1

Anterior Segment OCT images showing Ultra-Thin endothelial graft, before (a) and after (b) the rebubbling procedure. 